\title{
Formulation of Novel Glycerin Nanoparticles for Enhancement the Solubility of Loratadine: Application to Transdermal Hydrogel Delivery
} System

Heba A. Abou-Taleba1, Mohamed A. El Hamdb² and Ahmed A. H. Abdellatif ${ }^{*}$

${ }^{1}$ Department of Pharmaceutics and Industrial pharmacy, Faculty of Pharmacy, Nahda University, Benisuef, Egypt

${ }^{2}$ Department of Pharmaceutical Analytical Chemistry, Faculty of Pharmacy, Al Azhar University, Assiut, Egypt

${ }^{3}$ Department of Pharmaceutics and Industrial pharmacy, Faculty of Pharmacy, Al Azhar University, Egypt

\begin{abstract}
Owing to its slightly aqueous solubility, loratadine (LOR) is used in high doses in different marketed formulations to achieve its desirable bioavailability. The aim of this work is to formulate novel LOR-glycerin nanoparticles (LOR-GNPs) to increase LOR bioavailability. LOR-GNPs were prepared by a precipitation method and then evaluated for such size and morphology using dynamic scattering spectroscopy (DLS) and scanning electron microscopy (SEM), as well as the percentage entrapment efficiency (EE\%) of free LOR from GNPs formulations was performed using titration method. Furthermore, the GNPs were formulated in hydrogel. The hydrogel viscosity, spreadability, homogeneity and abdominal rat skin permeation were studied and optimized DLS indicated a successful coating of LOR with glycerin surface that recorded an average size of $334 \pm 30 \mathrm{~nm}$ with uniform particle size, while SEM showed LOR-NPs in different shapes. The EE\% free LOR was found to be satisfactory with a mean content of $98.1 \pm 0.3$ and a relative standard deviation below $2.0 \%$ indicates the reproducibility of the LOR release. LOR-GNPs were formulated as a hydrogel to check their suitability for a dosage form usage. The hydrogel showed accepted viscosity, spreadability, and homogeneity. The results proved that the GNPs penetrated abdominal rat skin. The obtained results showed that LOR-GNPs are considered a new addition for improvement of LOR solubility when applied as a hydrogel. The developed method could be used for different insoluble candidates.
\end{abstract}

Keywords: Loratadine; Glycerin; Nanoparticles; Precipitation method; Hydrogel

\section{Introduction}

An approximately $65 \%$ of the human body is made up of water, therefore drug molecules must have certain aqueous solubility to achieve an acceptable bioavailability level [1]. The aqueous solubility of drug molecules is a major challenge and critical step to achieve an optimal bioavailability after oral administration of its pharmaceutical dosage form. Loratadine (LOR, Figure 1) is a second-generation nonsedative tricyclic antihistaminic $\left(\mathrm{H}_{1}\right)$ drug. It is used to prevents the seasonal and perennial allergic rhinitis, allergic dermatitis, idiopathic urticaria and ocular allergy [2]. LOR belongs to biopharmaceutical classification system class II with low solubility and high permeation drug [3]. Accordingly, LOR is used in a high dose (10 mg/Tablet) to reach the desirable therapeutic blood concentration [4]. Unfortunately, the high doses of LOR affected its safety patient with renal and liver impairment. Through the literature, many methodologies have been reported to enhance the solubility of LOR; such as inclusion complex with $\beta$-cyclodextrins, spray-drying, freeze-drying and/or microwave irradiation [5-9]. Although these techniques were improved LOR solubility, but they are sophisticated, required a specialized equipment, time consuming and yield a poor flowing product powder [10]. Therefore, an effort was done to achieve novel simple, cost-effective LOR-glycerin nanoparticles (LOR-GNPs) to increase its solubility. The prepared nanoparticles were applied for a hydrogel dosage form using carbopol 934. The prepared hydrogel was optimized, evaluated, it's in vitro release and ex vivo skin permeation was checked to assess the transdermal ability to deliver its contents of LOR.

\section{Materials and Instrumentations}

\section{Materials}

The reference standard LOR powder was kindly supplied from

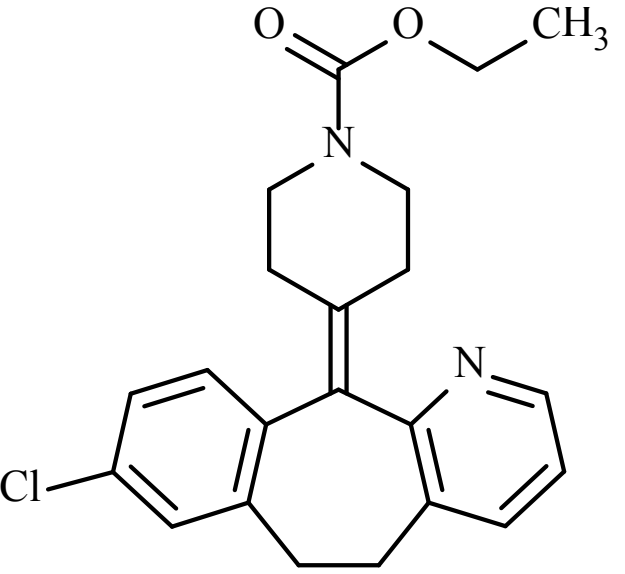

Figure 1: The chemical structure of loratadine.

*Corresponding author: Ahmed AH Abdellatif, Department of Pharmaceutics and Industrial pharmacy, Faculty of Pharmacy, Al Azhar University, Egypt, Tel: +20 1016660069; E-mail: ahmed.a.h.abdellatif@azhar.edu.eg

Received February 06, 2017; Accepted February 18, 2017; Published February 24, 2017

Citation: Abou-Taleb HA, Hamd MA, Abdellatif AAH (2017) Formulation of Nove Glycerin Nanoparticles for Enhancement the Solubility of Loratadine: Application to Transdermal Hydrogel Delivery System. J Nanomed Nanotechnol 8: 426. doi: 10.4172/2157-7439.1000426

Copyright: @ 2017 Abou-Taleb HA. This is an open-access article distributed under the terms of the Creative Commons Attribution License, which permits unrestricted use, distribution, and reproduction in any medium, provided the original author and source are credited. 
Medical Union Pharmaceuticals (MUP, Cairo, Egypt). Glycerin, carbopol 934 and methanol were purchased from Merck company (Darmstadt, Germany). Glacial acetic acid, perchloric acid $\left(\mathrm{HClO}_{4}\right.$, $70 \% \mathrm{w} / \mathrm{v})$, sodium hydroxide and crystal violet powder were obtained from El Nasr Chemical Co., (Abu Zaabal, Egypt). All other chemicals and reagents used through the study were of analytical reagent grade with distilled water was used throughout the work.

\section{Instrumentations}

A Sonicator model UH-100B (Tianjin Automatic Science Instrument Ltd, China), dynamic light scattering (DLS) and Malvern Zetasizer-Nano model 6.01 (Malvern instruments $\mathrm{GmbH}$, Herrenberg, Germany), scanning electron microscopy (SEM, Philips XL30, Netherlands), spindle No. 4 DV. Ultra, model RVDV-111U (Brookfield, USA), Rheometer (Maxwell Orthogonal Rheometer, USA), thermostatically controlled shaker model WSB-45 (DAIHAN Scientific Co., Korea), digital analytical balance model AG 29 (Mettler Toledo, Glattbrugg, Switzerland), digital pH meter model 3500 (Jenway, UK) and MLW type thermostatically controlled water bath (Memmert $\mathrm{GmbH}$, Schwabach, Germany) were used.

\section{Methods}

\section{Preparation of glycerin nanoparticles loaded with loratadine}

LOR-GNPs were prepared by precipitation method using LOR, glycerin, methanol, and distilled water. LOR, $20 \mathrm{mg}$ and glycerin, $5 \mathrm{~mL}$ were transfer into a beaker contains methanol, $5 \mathrm{~mL}$. The mixture was sonicated for 10 minutes and dropped into another beaker contains distilled water, $20 \mathrm{~mL}$ with continuous stirring at $500 \mathrm{rpm}$ using a magnetic stirrer and left overnight till evaporate the methanol phase. Then the final obtained LOR-GNPs were purified through filtration using a Whatman No. 42 filter paper to remove the large aggregates.

\section{Determination of size, count rate and polydispersity index} and zeta potential

DLS instrument was used to determine the size, count rate and polydispersity index (PDI) of non-medicated GNPs (blank) and medicated LOR-GNPs samples [11,12]. Two-milliliters of each sample were measured in a disposable cuvette in three experimental replicates. Each sample was checked by laser light instrument with an incident laser beam of $633 \mathrm{~nm}$ and a scattering angle of $90^{\circ}[13,14]$ at $25^{\circ} \mathrm{C}$. As well as, Zeta potential measurements were done by a Zetasizer instrument, using a standard zeta cuvette in three experimental replicates.

\section{Determination of surface morphology}

The surface morphology of blank GNPs and LOR-GNPs were determined using SEM instrument operated at 4-25 Kv on samples gold-sputtered for $120 \mathrm{~s}$ at $10 \mathrm{~mA}$ under argon gas and low pressure [15].

\section{Determination of entrapment efficiency\%}

The entrapment efficiency percent (EE\%) of LOR-GNPs was determined using a direct non-aqueous titration method [16], was developed and validated by the authors. Briefly, the formed LOR-GNPs were evaporated carefully on a hot plate until dryness. The residue was reconstituted in an appropriate volume of glacial acetic acid. Two drops of $0.1 \% \mathrm{w} / \mathrm{v}$ crystal violet indicator were added and the solution was titrated against a standard solution of $0.1 \mathrm{~N}$ perchloric acid to a pure blue end point. Then the $\%$ of LOR in LOR-GNPs calculated as the following:
Each $1 \mathrm{~mL}$ of $0.1 \mathrm{~N}$ perchloric acid $\equiv 0.0038 \mathrm{~g}$ LOR.

LOR $\%, w / v=($ End point $\times$ milliequivalent $\times 100) /($ weight of sample $)$

\section{Preparation of hydrogel loaded loratadine nanoparticles}

Carbopol 934 hydrogels were prepared by previous reported methods [13,17]. Briefly, carbopol $934(1 \%, w / v)$ was added gradually to a beaker contains an aqueous solution of LOR-GNPs with continuous stirring and heating in a water bath at $50^{\circ} \mathrm{C}$ until homogenous gel is formed. Then sodium hydroxide solution $(0.4 \%, \mathrm{w} / \mathrm{v})$ was added just to neutralize the free acid liberated from carbopol 934.

\section{Evaluation of hydrogel}

Determination of $\mathrm{pH}$ and entrapment entrapping efficiency\%: The $\mathrm{pH}$ values of a blank GNPs and LOR-GNPs hydrogel were measured using a digital $\mathrm{pH}$ meter. LOR-GNPs Hydrogel, $100 \mathrm{mg}$ was extracted by adding methanol, $100 \mathrm{~mL}$ into a beaker and sonicated for $5 \mathrm{~min}$. The supernatant were purified by filtration using a Whatman No. 42 filter paper. Then the EE\% of LOR content was determined by non-aqueous titration method as described in 3.4. [18].

Determination of rheological behavior and viscosity: A blank GNPs and LOR-GNPs hydrogel were evaluated for their rheological behavior by rheometer instrument using cone and plate configuration. The rheometer was equipped with cone-plate geometry (4/40) operating in the oscillation mode. The hydrogel samples were placed onto the bottom plate of the rheometer then the upper plate was then lowered to a gap size of $1000 \mu \mathrm{m}$.

The viscosity of the hydrogel was determined at different angular velocities at $25^{\circ} \mathrm{C}$ using spindle No. 4 . Viscosities were recorded at $25^{\circ} \mathrm{C}$ and $1 \mathrm{~Hz}$ oscillatory frequency as a function of the applied stress [13].

Determination of homogeneity and spreadability: The homogeneity of a blank GNPs and LOR-GNPs hydrogel were tested by visual appearance after a number of sample $(n=5)$ of the hydrogels have been introduced into their containers. Small quantity of each hydrogel sample is pressed between the thumb and the index finger to notice the consistency of the hydrogel whether homogeneous or not.

The spreadability was carried out for the gel formulation, by measuring diameter of $1 \mathrm{~g}$ gel among horizontal plates $\left(20 \times 20 \mathrm{~cm}^{2}\right)$ after 1 minute. The standardized weight fixed on the upper plate was $125 \mathrm{gm}$.

Determination of in vitro release: In vitro LOR release from LOR-GNPs hydrogel has been checked in a system resemble to Franz diffusion cell [13]. Briefly, cellophane membrane was stretched over the end of an open-ended glass tube and made water tight using a rubber band. The tube was immersed vertically in a $100-\mathrm{mL}$ beaker

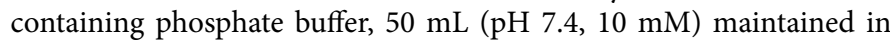
a thermostatically controlled shaker $\left(50\right.$ stroke/minute) at $37^{\circ} \mathrm{C}$. LORGNPs hydrogel equivalent to $20 \mathrm{mg}$ of LOR was placed into the glass tube. At predetermined time intervals for up to 24 hours, $5 \mathrm{~mL}$ aliquots of the release medium were withdrawn for analysis and were replaced with equal volume of phosphate buffer at the same temperature to maintain the volume constant. LOR content was determined using non-aqueous titration method as described in 3.4.

The data obtained from in vitro release studies were fitted to various kinetic models such as zero order, first order, and Higuchi's model in order to study the kinetics release [19]. Moreover, KorsmeyerPeppas model as the log logarithm of cumulative percentage of LOR released versus logarithm of time (log time) was used to determine the 
mechanism of LOR release. Then slope of the produced straight line could be used to calculate the value of the exponent (n). The relations were used to interpret the result outputs are:

a. $\quad \mathrm{n}=0.5$, the diffusion mechanism is fickian, as in the case with slab matrix system.

\section{b. $\quad 0.5<\mathrm{n}<1.0$, the diffusion mechanism is non-fickian. \\ c. $\mathrm{n}=1.0$, refers to a case II relaxation transport. \\ d. $\quad \mathrm{n}<1.0$, refers to super case II transport.}

Determination of the stability: By storing LOR-GNPs hydrogel at two different temperatures $4^{\circ} \mathrm{C}$ and $25^{\circ} \mathrm{C}$ for 30 days, the physical and chemical stability were investigated [18]. The physical stability was assessed by visual observation whatever any sedimentation detected or no or via particle size determination using a Zetasizer instrument. While the chemical stability was determined by measuring the free LOR content released from LOR-GNPs hydrogel using the non-aqueous titration method as described in 3.4

Ex vivo determination of the permeability of hydrogel loaded loratadine using abdominal skin of rat: The permeated or cumulative amount of LOR across rat abdominal skin treated with LOR-GNPs hydrogel after 12 hours was studied. A blank GNPs hydrogel and LOR-GNPs hydrogel were applied on two different groups of rats. The hydrogel was conveyed using abdominal male rat skin (weighed $140 \pm$ $20 \mathrm{~g}, \mathrm{n}=5$ ) [20]. The rats were first sacrificed and their hair was removed from the dorsal side of the rat using $0.1 \mathrm{~mm}$ hair clipper to develop the fresh skin of the rats. A wet cotton swab soaked in isopropanol and wipes the dermal part of the skin for any remaining fat materials. Skin turn out to be saturated with phosphate buffer before permeation study by soaking them in phosphate buffer for 6 hours. Skin portion was strained over one end of an open-ended glass tube, then immersed in a $400-\mathrm{mL}$ beaker holding $125 \mathrm{~mL}$ of the buffer and held in vertical situation. The membrane was just under the surface of the buffer solution. The tube (donor) and beaker (acceptor) were kept at $37^{\circ} \mathrm{C}$ in a thermostatically controlled shaker water bath. The donor partition was occupied with $0.25 \mathrm{~g}$ of hydrogel. At time breaks (up to 24 hours) samples of $2.0 \mathrm{~mL}$ were removed from the receptor and replaced with a phosphate buffer [13]. Then the mean of three experiment replicates using non-aqueous titration method to determine the free LOR content were performed.

The permeability coefficient $\left(\mathrm{K}_{\mathrm{p}}\right)$ of LOR across the abdominal rat skin was estimated using Fick's first law of diffusion and expressed by the following equation: $K_{p}=\frac{J}{C}$

Where, $J$ is the flux ( $\mathrm{mg} / \mathrm{cm}^{2} /$ hours $)$ and $C$ is the LOR-concentration in donor partition.

The significant difference statistically with done by ANOVA/Tukey tests at probability $(p)<0.05$.

\section{Result and Discussion}

Glycerol a polyol compound gives it a significant positive effect on the properties of some soluble and insoluble agents [21]. In this study, glycerol was utilized a coating material to enhance the aqueous solubility of slightly soluble LOR using a precipitation method to prepare LORGNPs by the temporary aid of methanol. LOR-glycerin in a mixture with methanol was added to an aqueous solution with a vigorous stirring. The solution initially, is clear and colorless then changed into cloudy solution indicated that the solution containing highly dispersed nanoparticles containing LOR-glycerin. Desirable size, zeta potential,
PDI and adjusting the surface charge are parameters that indicate the colloidal stability of the obtained nanoparticles system [13-14,22-24].

The results showed an acceptable small and uniform LOR-GNPs particle size. Figure 2 shows the blank GNPs and formulated LORGNPs were of a uniform $Z$ average diameter of $334 \pm 76 \mathrm{~nm}$. DLS recorded only one peak with intensity of $100 \%$. The blank GNPs had a $\mathrm{Z}$ average diameter of $1403 \pm 20 \mathrm{~nm}$ and recorded two peaks; peak (a) at $277.1 \mathrm{~nm}$ with intensity of $57.9 \%$ and peak (b) at $1.3 \mathrm{~nm}$ with intensity $42.1 \%$. PDI is a measure of the distribution of molecular mass in a given polymer sample an also index that could indicate of the stability of the LOR-GNPs since it represents the particles distribution in colloidal solution. High PDI values indicates the heterogeneity of the particle size in suspension, while smaller PDI indicate the homogeneity of the particle size in suspension, ideally the value of PDI should be $<0.7$ because they indicate a particle size distribution falls within a narrow range of sizes [22,25-28]. In the present study the PDI average value of $\approx$ $0.3 \pm 0.02$ was recorded for the formulated GNPs-LOR which revealed the homogeneity of the particle size in the obtained suspension. The high PDI average value of 1.0 was recorded by DLS for blank GNPs indicates the heterogeneity of the particle size in suspension. These results confirmed that LOR-glycerin and LOR-GNPs were highly water soluble, while the blank GNPs have not the same characteristics of LORGNPs. Furthermore the PDI was very small indicating the uniformity of the obtained LOR-GNPs and their mono-dispersity related by the perfectly systematic size distributions curves as showed in Figure 2. Furthermore, these results confirmed that the glycerin coated the LOR, and hence LOR was nucleus for nanoparticles.

Zeta potentials were measured to confirm the successful coating of LOR with glycerin. Zeta potential of all particle types was initially negative. Then after coating with glycerin it became more positive, Figure 3, indicated the successful coating of LOR with glycerin. Before deposition of glycerin, LOR had negative zeta potential of $\approx-20.8 \pm$ 2.7 and after coating the LOR the surface of LOR-GNPs had positive surface zeta potentials of $+43.7 \pm 3.2$. The high-count rate $(185 \mathrm{kpcs})$ indicates that the concentration of nanoparticles was high enough for measurements.

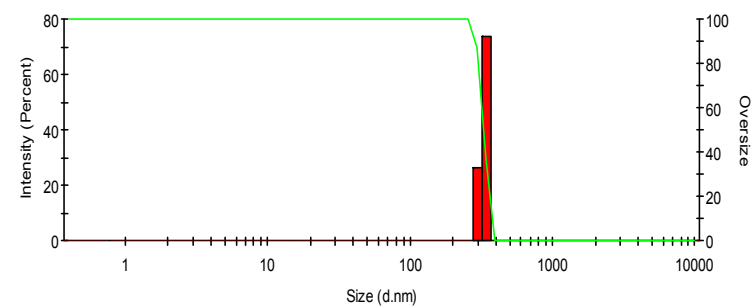

b

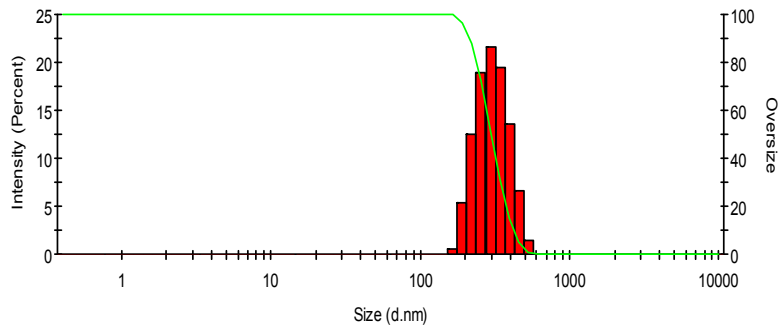

Figure 2: Particle size distribution of GNPs measured using dynamic ligh scattering, shape of blank GNPs (a), as glycerin solution dropped on wate which showed no uniformity of nanoparticles while LOR-GNPs (b) which showed uniform and monodisperse GNPs. 
Citation: Abou-Taleb HA, Hamd MA, Abdellatif AAH (2017) Formulation of Novel Glycerin Nanoparticles for Enhancement the Solubility of Loratadine: Application to Transdermal Hydrogel Delivery System. J Nanomed Nanotechnol 8: 426. doi: 10.4172/2157-7439.1000426

Page 4 of 6

In order to prove the formulation of GNPs-LOR, the blank and GNPs-LOR were examined by SEM. While the blank of GNPs showed non-identified nanoparticles, GNPs-LOR were identified and showed as in Figure 4a. The formulated LOR-GNPs were homogeneous and free of aggregates. They are present in different shapes such as cubes, rods, or triangles, (Figure $4 \mathrm{~b}$ ). These shapes were also as combination between two or three GNPs-LOR. The surface of the LOR-GNPs was slightly rough due to the higher concentration of drug that uniformly dispersed at the molecular level.

Figure $4 \mathrm{~b}$ shows the typical results of the studies of LOR-GNPs deposited on a carbon strip by means of SEM. These results don't interfere with the results obtained from DLS, as the DLS showed monodisperse nanoparticles with single peak. Otherwise, these nanoparticles were dried then were examined by SEM which showed different shapes for nanoparticles staked together.

The results of $\mathrm{pH}$, viscosity, spreadability, homogeneity, and drug content studies are presented in Table 1. All blank GNPs, LOR-GNPs and LOR-GNPs hydrogel have a $\mathrm{pH}$ values similar to the abdominal rat skin value which indicated the compatibility of these formulations with the skin of rat. The prepared LOR-GNPs hydrogel showed a high viscosity values compared to blank GNPs hydrogel which made this formulation more suitable for transdermal delivery. Moreover, it showed a higher spreadability value of $6.5 \pm 0.8 \mathrm{~g} . \mathrm{cm} / \mathrm{second}$ than those of blank hydrogel (of $5.5 \pm 0.7 \mathrm{~g} . \mathrm{cm} / \mathrm{second}$ ). On the other hand, the blank GNPs, LOR-GNPs, and LOR-GNPs hydrogel had a good homogeneity with absences of any lumps as they were uniform in consistency and free from any appreciable particulate matter after their microscopic examination. The Entrapping Efficiency of LOR content in the formed hydrogel was found to be satisfactory with a mean content of $98.1 \pm 0.3$ with a relative standard deviation below $2.0 \%$ indicates
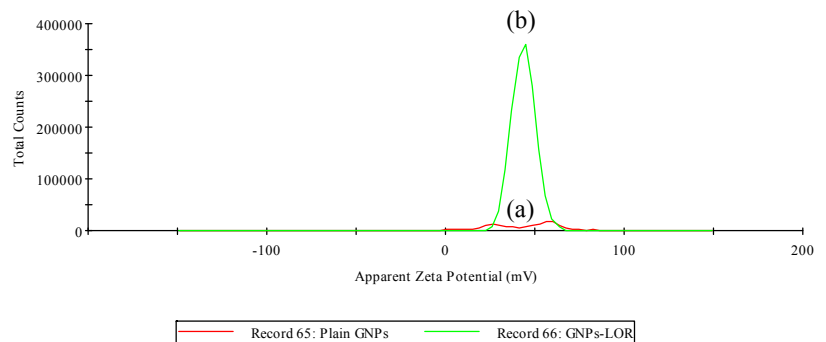

Figure 3: Zeta potential peaks of blank (a) and LOR-GNPs (b) GNPs measured using dynamic light scattering of glycerin solution dropped on.
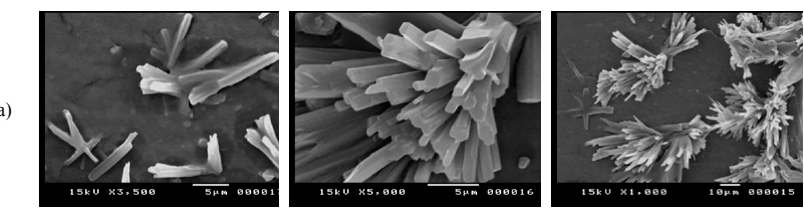

(b)
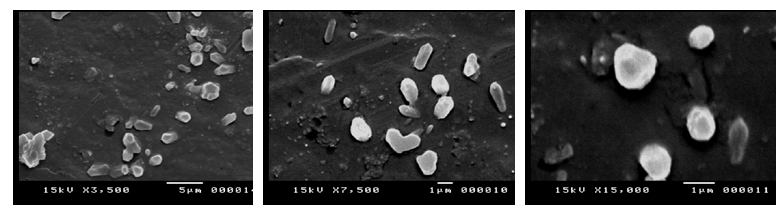

Figure 4: Scanning electron microscope images of blank GNPs and LORGNPs. The view of blank GNPs at $3.500 \times 5.000$ and 1.000 magnification respectively, (a). The view of the LOR-GNPs at 3.500, 7.500 and 15.000 magnifications respectively, (b)

\begin{tabular}{|c|c|c|c|c|c|}
\hline Formulation & $\mathbf{p H}$ & Viscosity & Spreadability & Homogeneity & $\begin{array}{c}\text { Entrapping } \\
\text { Efficiency }\end{array}$ \\
\hline Blank GNPs & $6.8 \pm 0.3$ & $4260 \pm 65$ & $5.5 \pm 0.7$ & good & Nil \\
\hline LOR-GNPs & $7.1 \pm 0.4$ & - & - & good & $98.8 \pm 3.9$ \\
\hline $\begin{array}{c}\text { LOR-GNPs } \\
\text { hydrogel }\end{array}$ & $6.9 \pm 0.2$ & $4630 \pm 33$ & $6.5 \pm 0.8$ & good & $96.4 \pm 3.9$ \\
\hline
\end{tabular}

Table 1: $\mathrm{pH}$, viscosity (cps), spreadability (g.cm/second), homogeneity and drug content (\%) of blank GNPs and LOR-GNPs hydrogel. $\mathrm{N}=3 \pm$ standard deviation (SD).

the reproducibility of the LOR concentration in the hydrogel, hence the method adopted for hydrogel formulation.

To evaluate the LOR-GNPs hydrogel release, it was subjected to in vitro drug release studies using a cellophane membrane as illustrated in Figure 5. The cumulative amounts of LOR released were calculated from LOR-Gel, which showed a significant higher LOR release $(\mathrm{p}<0.05$, ANOVA/Tukey tests) from the LOR-GNPs hydrogel of $79.1 \pm 1.6 \%$ after $8 \mathrm{hr}$. The acceptable LOR release from LOR-GNPs hydrogel could be attributed to the presence of glycerin molecule in this formulation which has a higher solubility in phosphate buffer.

Table 2 shows blank GNPs and LOR-GNPs hydrogel, were best fitted for the Higuchi kinetic equation as the coefficient of correlation (r) values predominates over zero and first order kinetics. These results indicate that LOR permeation mechanisms for both formulations were by diffusion or slow or sustained release from the membrane. The best fitting model was $n$ value of $1.05 \pm 0.02$, which $>1.0$ indicates super case II Korsmeyer-Peppas model. This was confirming that LOR-release is controlled mainly by diffusion mechanism. Also confirmed, the release kinetics of LOR-GNPs hydrogel could be acted as reservoir systems for continuous delivery of the dispersed drugs.

The physical stability of LOR-GNPs and LOR-GNPs hydrogel was performed at two different conditions of $4{ }^{\circ} \mathrm{C}$ and $25^{\circ} \mathrm{C}$ for 30 days. The results of this study showed no sedimentation in any vesicle formulation after fresh preparation and after storage either at $4^{\circ} \mathrm{C}$ or room temperature. Furthermore, particle size analysis did not show any significant difference than those of fresh samples.

Nearly, the in vitro permeation results give valuable information about the applicability of LOR-GNPs hydrogel product in vivo (Figure 6). These results suggested the amounts of LOR (in the hydrogel preparation) could present to give the desirable amounts for its absorption across the abdominal skin of rat.

The average of the permeated amounts of LOR from GNPs and from hydrogel across the abdominal rat skin after 12 hours was $2.9 \pm$ $0.1 \mu \mathrm{g} / \mathrm{cm}^{2}$. This value average is significantly different than the blank GNPs hydrogel which is $3.7 \pm 0.2 \mu \mathrm{g} / \mathrm{cm}^{2}$. The mean values $(\mathrm{n}=3)$ of the steady state flux and permeability co-efficient was presented in Table 3 , showing the mean of the steady state flux and permeability co-efficient ranged from $3.7 \pm 0.9 \times 10^{-2}$ to $13.9 \pm 0.8 \times 10^{-2} \mu \mathrm{g} / \mathrm{cm}^{2} /$ hours, for blank GNPs and LOR-GNPs hydrogel respectively. The higher permeability of the LOR-GNPs hydrogel may be attributed to partitioning of vesicles into the stratum corneum, which is an important process as it drives the partitioning of vesicle-bound drug into the skin. The better partitioning of LOR-GNPs hydrogel with the stratum corneum and in the deeper layer of skin under the influence of the transepidermal osmotic gradient could be considered one of the reasons for the better skin permeation of LOR-GNPs [29]. The above hypothesis was well supported by Kirjavainen et al. [30] whose demonstrated that phospholipids affect the stratum corneum lipid bilayer fluidity and improve drug partitioning into the bilayer. 


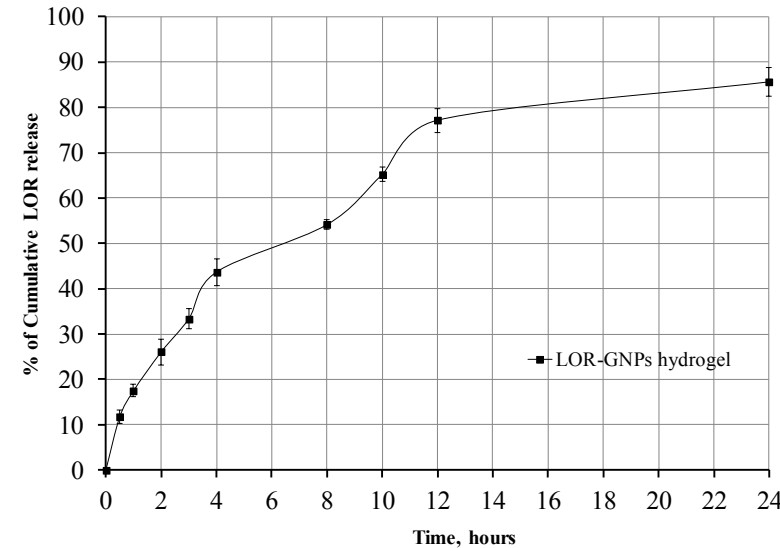

Figure 5: In vitro\% of LOR-GNPs hydrogel release using cellophane membrane.

\begin{tabular}{|c|c|c|c|c|}
\hline Formulation & $\begin{array}{c}\text { Zero order } \\
\text { model } \mathbf{( r )}\end{array}$ & $\begin{array}{c}\text { First order } \\
\text { model } \mathbf{( r )}\end{array}$ & $\begin{array}{c}\text { Higuchi } \\
\text { model } \\
\mathbf{( r )}\end{array}$ & $\begin{array}{c}\text { Korsmeyer- } \\
\text { Peppes Model } \\
\mathbf{( n )}\end{array}$ \\
\hline Blank GNPs & $0.921 \pm 0.028$ & $-0.156 \pm 0.016$ & $\begin{array}{c}0.989 \pm \\
0.007\end{array}$ & $1.120 \pm 0.050$ \\
\hline $\begin{array}{c}\text { LOR-GNPs } \\
\text { hydrogel }\end{array}$ & $0.904 \pm 0.016$ & $-0.128 \pm 0.027$ & $\begin{array}{c}0.983 \pm \\
0.006\end{array}$ & $1.050 \pm 0.020$ \\
\hline
\end{tabular}

Table 2: Zero, First, Higuchi models values and Korsmeyer-Peppes model ( $n$ ) for LOR released from LOR-GNPs hydrogel. $\mathrm{N}=3 \pm \mathrm{SD}$.

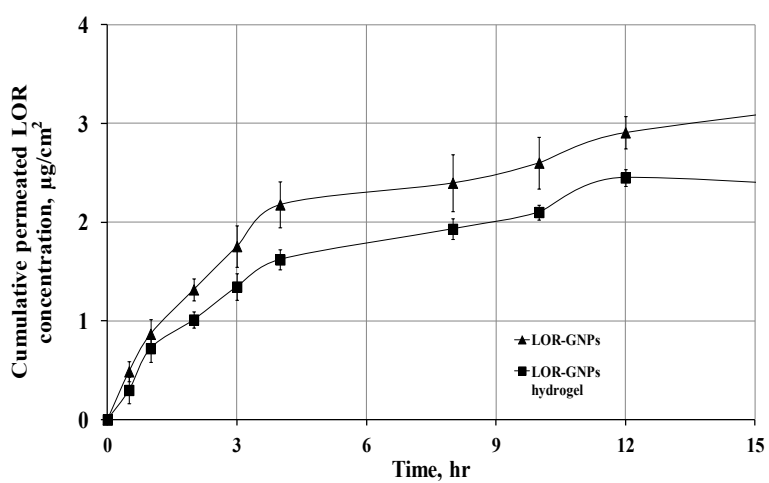

Figure 6: In vitro permeation studies of LOR-GNPs hydrogel as parameters which indicate the amount of LOR available for absorption.

\begin{tabular}{|c|c|c|c|}
\hline Formulation & $\begin{array}{c}\text { LOR permeated } \\
\text { concentration, at } 12 \\
\text { hours }\left(\mu \mathrm{g} / \mathrm{cm}^{2}\right)\end{array}$ & $\begin{array}{c}\left.\text { Flux (mg/cm }{ }^{2} / \text { hour }\right) \\
\times 10^{-2}\end{array}$ & $\begin{array}{c}\text { Permeability } \\
\text { coefficient (kp) } \times \\
10^{-3} \text { (cm/hour) }\end{array}$ \\
\hline $\begin{array}{c}\text { Blank GNPs } \\
\text { hydrogel }\end{array}$ & $1.6 \pm 0.1$ & $3.6 \pm 0.9$ & $10.5 \pm 1.1$ \\
\hline $\begin{array}{l}\text { LOR-GNPs } \\
\text { hydrogel }\end{array}$ & $2.7 \pm 0.2$ & $13.9 \pm 0.8$ & $36.5 \pm 2.6$ \\
\hline
\end{tabular}

Table 3: Concentration of free LOR permeated at 24 hours. $N=3 \pm S D$.

\section{Conclusion}

A novel simple and rapid method for LOR-GNPs preparation was developed and carefully evaluated to enhance the solubility of LOR in its pharmaceutical preparations. Moreover the applicability of this method was checked in a new LOR-GNPs hydrogel. Different studies were performed to indicate that the prepared LOR-GNPs hydrogel has a good in vitro release and permeability. Next, further preclinical studies in vivo are needed to confirm that the bioavailability of LOR is improved too following enhancement of its solubility. The prepared LOR-GNPs could be used in the preparation of LOR different dosage forms.

\section{References}

1. Yalkowsky S (1981) Techniques of solubilization of drugs. New York: Marcel Dekker.

2. Moffat A, Osselton K, Widdop L (2004) Clarke's Analysis of Drugs and Poisons (3rdedn) London.

3. Khan MZI, Rausal D, Zanoski R, Zidar S, Mikulci JH, et al. (2004) Classification of loratadine based on the biopharmaceutics drug classification concept and possible in vitro-in vivo correlation. Biol Pharm Bull 27: 1630-1635.

4. Mitali K, Lin L, Rainer HM (2012) Overcoming the challenge of poor drug solubility. Pharmaceutical Engineering 32: 1-7.

5. Lin SY, Lin HL, Lin CC, Hsu CH, Wu TK (2011) Thermodynamic study of grinding-induced loratadine inclusion complex formation using thermal analysis and curve-fitted FTIR determination. Thermodynamics - Physical Chemistry of Aqueous Systems.

6. Ambrus N, Berkesi R, Szabó-Révész O, Aigner PZ (2008) Water-soluble loratadine inclusion complex: analytical control of the preparation by microwave irradiation. Journal of Pharmaceutical and Biomedical Analysis 48: 1020-1023.

7. Omar L, El-Barghouthi MI, Masoud NA, Abdoh AA, Al Omari, et al. (2007) Inclusion complexation of loratadine with natural and modified cyclodextrins: Phase solubility and thermodynamic studies. Journal of Solution Chemistry 36 : 605-616.

8. Nacsa A, Berkesi O, Szabó-Révész P, Aigner Z (2009) Achievement of pH independence of poorly-soluble, ionizable loratadine by inclusion complex formation with dimethyl- $\beta$-cyclodextrin. Journal of Inclusion Phenomena and Macrocyclic Chemistry 64: 249-254.

9. Lin SY, Hsu CH, Sheu MT (2010) Curve-fitting FTIR studies of loratadine/ hydroxypropyl-beta-cyclodextrin Inclusion complex Induced by co-grinding process. Journal of Pharmaceutical and Biomedical Analysis 53: 799-803.

10. Ketan TS, Anuradha KG, Jignasa KS (2012) Drug solubility: Importance and enhancement techniques. International Scholarly Research Network ISRN Pharmaceutics.

11. Abdellatif A, El Rasoul SA, Osman S (2015) Gold nanoparticles decorated with octreotide for somatostatin receptors targeting. Int J Pharm Sci Res 7: 14-20.

12. Abdellatif A, Zayed G, El-Bakry A, Zaky A, Saleem IY, et al. (2016) Novel gold nanoparticles coated with somatostatin as a potential delivery system for targeting somatostatin receptors. Drug Dev Ind Pharm 42: 1782-1791.

13. Abdellatif A, Tawfeek HM (2016) Transfersomal nanoparticles for enhanced transdermal delivery of clindamycin. AAPS PharmSciTech 17: 1067-1074.

14. Abdellatif $A$ (2015) Targeting of somatostatin receptors using quantum dots nanoparticles decorated with octreotide. Journal of Nanomedicine and Nanotechnology S6: 1-8.

15. El-Zahry MR, Mahmoud A, Refaat IH, Mohamed HA, Bohlmann H, et al. (2015) Antibacterial effect of various shapes of silver nanoparticles monitored by SERS. Talanta 138: 183-189.

16. Mohamed EH, Ramadan A, Abdellatif AA (2016) Non-aqueous titrimetric assay for determination of loratadine in pharmaceutical preparations. Analytical and Bioanalytical Techniques 7: 1 - 3.

17. Abdellatif A, Tawfeek $H$ (2016) Metformin Loaded Carbopol Gel for lowering the Intra-Abdominal Visceral Fat. J Bioequiv Availab 8: 149-152.

18. Abdellatif A, Tawfeek HM (2015) Transfersomal Nanoparticles for Enhanced Transdermal Delivery of Clindamycin. AAPS PharmSciTech.

19. El Hamd AM, Saleh K (2016) A Formulation, Optimization and Evaluation of Controlled Released Alginate Beads Loaded-Flurbiprofen. Journal of Nanomedicine and Nanotechnology.

20. Pillai O, Panchagnula R (2003) Transdermal delivery of insulin from poloxamer gel: ex vivo and in vivo skin permeation studies in rat using iontophoresis and chemical enhancers. J Control Release 89: 127-140.

21. Jiang X, Zhao Y, Hou L (2016) The effect of glycerol on properties of chitosan/ 
Citation: Abou-Taleb HA, Hamd MA, Abdellatif AAH (2017) Formulation of Novel Glycerin Nanoparticles for Enhancement the Solubility of Loratadine: Application to Transdermal Hydrogel Delivery System. J Nanomed Nanotechnol 8: 426. doi: 10.4172/2157-7439.1000426

poly(vinyl alcohol) films with $\mathrm{AICl} 3.6 \mathrm{H} 2 \mathrm{O}$ aqueous solution as the solvent for chitosan. Carbohydrate Polymers 135: 191-198.

22. Pereira-Lachataignerais J, Pons R, Panizza P, Courbin L, Rouch J, et al. (2006) Study and formation of vesicle systems with low polydispersity index by ultrasound method. Chem Phys Lipids. Chemistry and Physics of Lipids 140: 88-97.

23. Moraes CM, De Paula E, Rosa A, Fraceto L (2010) Physicochemical stability of poly(lactide-co-glycolide) nanocapsules containing the local anesthetic bupivacaine. Journal of the Brazilian Chemical Society 21: 995-1000.

24. Kaufman E, Belyea J, Johnson M, Nicholson Z, Ricks J, et al. (2007) Probing protein adsorption onto mercaptoundecanoic acid stabilized gold nanoparticles and surfaces by quartz crystal microbalance and zeta-potential measurements. Langmuir 22: 6053-6062.

25. Aghajani M, Shahverdi A, Amani A (2012) The use of artificial neural networks for optimizing polydispersity index (PDI) in nanoprecipitation process of acetaminophen in microfluidic devices. AAPS PharmSciTech.
26. Lewis G, Li Y (2010) Dependence of in vitro fatigue properties of PMMA bone cement on the polydispersity index of its powder. Journal of the Mechanical Behavior of Biomedical Materials 3: 94-101.

27. Rosenfeld C, Serra C, Brochon C, Hadziioannou G (2008) Influence of micromixer characteristics on polydispersity index of block copolymers synthesized in continuous flow microreactors. Lab on a Chip 8: 1682-1687.

28. Vieville J, Tanty M, Delsuc M (2011) Polydispersity index of polymers revealed by DOSY NMR. Journal of Magnetic Resonance 212: 169-173.

29. Gupta A, Aggarwal G, Singla S, Arora R (2012) Transfersomes: a nove vesicular carrier for enhanced transdermal delivery of sertraline: development, characterization, and performance evaluation. Scientia pharmaceutica 80 1061-1080.

30. Kirjavainen M, Monkkonen J, Saukkosaari M, Valjakka-Koskela R, Kiesvaara $\mathrm{J}$, et al. (1999) Phospholipids affect stratum corneum lipid bilayer fluidity and drug partitioning into the bilayers. Journal of Controlled Release 58: 207-214. 